\title{
Relationship between fragmented QRS and microvascular function in patients with inferior myocardial infarction
}

\author{
Mehmet Zülkif Karahan ${ }^{1}$, Bernas Altintas ${ }^{2}$, Önder Öztürk ${ }^{2}$, Mehmet Sahin Adiyaman², \\ Burhan Aslan² ${ }^{2}$ İlyas Kaya ${ }^{2}$, Önder Bilge ${ }^{2}$, Abdurrahman Akyüz ${ }^{2}$, and Sedat Kaya ${ }^{2}$ \\ ${ }^{1}$ Diyarbakir Gazi Yasargil Training and Research Hospital \\ ${ }^{2}$ Affiliation not available
}

November 13, 2020

\begin{abstract}
Abstract Objective: The purpose of this study is to investigate the relationship between microvascular dysfunction and the presence of fragmented QRS(f-QRS) in patients with acute inferior myocardial infarction(MI) who underwent primary percutaneous coronary intervention(PPCI). Methodology:274 consecutive patients with a mean age of $56.8 \pm 9.8$ who met the inclusion criteria were enrolled; patients with TIMI 2-3 flow after PPCI were divided into two groups according to the myocardial blush grade (MBG) 0-1 and MBG 2-3. The f-QRS includes different morphologies of the QRS and includes an additional $\mathrm{R}$ wave $(\mathrm{R}$ ') or notching at the lowest end of the $\mathrm{S}$ wave in two adjacent leads releasing the infarct area. ECG records were taken to assess f-QRS and ST segment resolution was assessed in the first hour after the procedure. During angiographic examination, myocardial blush grade (MBG) and TIMI flow were measured in the right coronary artery due to post-procedure infarction. Results:The patients were divided into two groups as MBG 0-1 and MBG 2-3. In general, the median age was 56.8 \pm 9.8 ,and 49 patients(17.9\%) were women. Among all study patients, f-QRS count was 36(13.1\%). In this study, 62 and 212 patients had MBG 0-1 and MBG 2-3, respectively. f-QRS was detected in 23(21.7\%) patients in the MBG 0-1 group and 13(10.7\%) patients in the MBG 2-3 group. In multivariate logistic regression, f-QRS [OR: 2.3(1.13-5.06),p =0.027],ST segment resolution at first hour [OR: $0.62(0.39-0.90), \mathrm{p}=0.04]$, and TIMI frame number [OR: $1.05(1.01-1.09), \mathrm{p}=0.004$ ] were found to be associated with MBG 0-1. Conclusion:Our study showed that the presence of f-QRS after PPCI was associated with microvascular dysfunction in patients with inferior MI who underwent successful PPCI.F-QRS, a simple and inexpensive parameter, can be used to assess microvascular dysfunction in MI patients who underwent PPCI. Keywords:microvascular dysfunction,fragmented QRS,myocardial infarction
\end{abstract}

\section{Introduction}

Acute myocardial infarction (AMI) is an important cause of morbidity and mortality in patients with coronary heart disease worldwide. It is important to identify high-risk patients, and the main goal of treatment in AMI is to restore microvascular flow and maintain myocardial perfusion (1). Various markers such as electrocardiography and coronary angiography have been used to determine myocardial reperfusion $(2,3)$.

Fragmented QRS in 12-lead ECG has been shown to be associated with myocardial scarring in heart disease $(4,5)$ and it is also associated with cardiac mortality and morbidity in heart disease $(6,7)$. The presence of f-QRS has been associated with predicting ventricular tachyarrhythmia in ischemic cardiomyopathy, nonischemic cardiomyopathy, hypertrophic obstructive cardiomyopathy, Brugada syndrome, and arrhythmogenic right ventricular cardiomyopathy, and it has been also associated with sudden cardiac death in these diseases (8).

Although f-QRS is associated with depolarization, it is so far poorly understood regarding microvascular function in patients with acute myocardial infarction. The purpose of this study is to assess the relationship 
between microvascular dysfunction and the presence of f-QRS in patients with acute inferior myocardial infarction (MI) who underwent successful primary percutaneous coronary intervention (PPCI).

\section{Patients and Methodology}

306 consecutive patients diagnosed with acute inferior MI were enrolled in this study after discharge following the review of their records. MI was diagnosed according to ACC / AHA 2012 guidelines. 32 patients were excluded due to problems in the follow-up process. In addition, patients who had a heart attack and had a previous heart attack were not included in the study. The remaining 274 patients with a median age of 56.8 \pm 9.8 years and with TIMI 2-3 after PPCI were included in the study. The patients were divided into two groups as MBG 0-1 and MBG 2-3.

ECG records were taken to assess the fragmented QRS and ST segment resolution was assessed in the first hour ECG routinely taken after the procedure. A 12-lead ECG with a paper speed of $25 \mathrm{~mm} / \mathrm{sec}$ was recorded in each patient at admission and 60 minutes after primary PCI. The fragmented QRS includes different morphologies of the QRS, and the $\mathrm{S}$ wave in two adjacent leads contains an additional $\mathrm{R}$ wave $(\mathrm{R}$ ') or notching at the lowest end of the damaged area. The presence of fragmented QRS in 2 adjacent inferior leads (II, III and aVF) indicated myocardial scar and necrosis in the inferior region. For ST segment resolution, the largest ST segment elevation on the baseline ECG was taken as a reference for subsequent assessment of ST segment elevation. ST segment measurement was made 60 milliseconds after J point at the ST segment point. QRS duration was calculated manually according to the clinical condition of the patients and was measured from the earliest beginning to the end of the last QRS deflection. QT was defined as the interval between the beginning of the QRS and the end of the T wave. QT dispersion was calculated as the maximum QT interval minus the minimum QT interval. All ECGs were analyzed manually by a single experienced cardiologist who was blinded to this study.

Blood samples were taken from all patients and measurements were made using a standard Beckman Coulter LH 780 Analyzer.

All patients were given $300 \mathrm{mg}$ aspirin and $600 \mathrm{mg}$ loading dose of clopidogrel before the procedure, and intravenous heparin 10,000 $\mathrm{U}$ and intracoronary nitroglycerin were administered at the beginning of the procedure. The use of glycoprotein IIb / IIIa inhibitor (tirofiban) was at the discretion of the operator. First balloon angioplasty and then coronary artery stenting were performed in all patients.

All coronary angiographies were recorded using Philips AlluraXper FD 10/10 device. First balloon angioplasty and then coronary artery stenting were performed in all patients. The contrast injector was set at 4 $\mathrm{mL} / \mathrm{sec}$ for the left coronary artery $(8 \mathrm{~mL}$ bolus) and $3 \mathrm{~mL} / \mathrm{sec}$ for the right coronary artery (6 mL bolus). MBG was used to assess myocardial blush during angiography (9). Myocardial blush grading was performed visually in a catheterization lab with a sinus film at a rate of $25 \mathrm{fps}$. This should have been sufficient to assess the length of the coronary angiography. In this study, coronary angiography was sufficient to see the venous phase of the contrast passage, and coronary angiography was performed with the same images as the infarct-related artery. In the assessment of MBG, a single image was selected from multiple orthogonal views to minimize overlapping of non-infarction areas. In most of the patients, right anterior oblique (RAO) was used for the right coronary artery, and laterolateral or RAO was used for the circumflex coronary artery. Two experienced cardiologists blinded to the study performed the MBG classification. The reproducibility of MBG was assessed by 2 observers who examined 40 coronary angiograms. Inter-observer and intra-observer agreement was $90 \%$ and $90 \%$, respectively. TIMI flow grades were assessed as previously described (10) and coronary atherosclerosis density was assessed using the Gensini score (11).

Echocardiographic evaluation using the Hewlett Packard SONOS 4500 and 2.5 to $3.5 \mathrm{mHz}$ transducer according to the American Echocardiographic Association criteria was performed within the first 24 hours after primary PCI by two cardiologists blinded to this study. Left ventricular ejection fraction (EF) was measured using the modified Simpson method. The study was approved by the local Ethics Committee.

\section{Statistical Analyses}


SPSS 18.0 (SPSS, Inc, Chicago, Illinois) was used statistically for analysis. Kolmogorov-Smirnov test evaluated the distribution of continuous variables. Student's t test for continuous variables was used to analyze the differences between main groups. In addition, univariate and multivariate logistic regression analyzes were performed to evaluate the relationship between myocardial blushing grade and fragmented QRS, ST segment resolution, and TIMI frame count. In the stepwise multivariate regression analysis (Backward, Wald), the effect size was adjusted for all variables with a univariate significance level of $<0.2$. Adjusted odds ratios (OR) are presented with $95 \%$ CIs. Continuous variables were expressed as mean + standard deviation and categorical variables were expressed as percentages. Two-tailed $\mathrm{P}$ values $<0.05$ were considered statistically significant.

\section{Results}

From January 2017 to December 2019, 306 consecutive patients diagnosed with acute inferior MI were enrolled in this study after discharge following the review of their records. The number of patients excluded during the follow-up period and reasons for exclusion were as follows: 23 patients $(0.07 \%)$ were excluded due to TIMI 0-1 flow, 7 patients $(0.02 \%)$ due to bundle branch blocks, and 2 patients $(0.006 \%)$ due to chronic renal failure. A total of 274 patients (median age $56.8 \pm 9.8$ years) who presented with the first acute inferior myocardial infarction and whose infarct-related artery was the right coronary artery were included in the study. Primary percutaneous coronary intervention (PPCI) was performed in all patients. According to patients with TIMI 2-3 flow after PPCI, patients were divided into two groups of MBG 0-1 and MBG 2-3. In this study, 62 and 212 patients had MBG 0-1 and MBG 2-3, respectively. The demographics and laboratory parameters of the patients are shown in Table 1. As shown in Table 1, no significant difference was found between the two groups in demographics and clinical characteristics. Compared with the MBG 2-3 groups, the MBG 0-1 groups had a significantly lower left ventricular ejection fraction and higher white blood count, and the differences were statistically significant.

Comparison of angiographic and electrographic parameters between groups is shown in Table 2. Fragmented QRS was more pronounced in patients with impaired microvascular reperfusion. MBG 2-3 groups and MBG $0-1$ groups were similar in pain-to-balloon time $(4.64 \pm 1.84$ vs. $57 \pm 1.8$, p: 0.78$)$. Compared with the MBG 2-3 groups, the ST segment resolution at one hour was significantly lower and the QRS duration was significantly higher in the MBG 0-1 groups. However, the TIMI frame count was higher in the MBG 0-1 groups. In this study, there was no statistically significant difference between the two groups and between the severity of coronary artery disease according the Gensini score. However, the Gensini scores were high in the patients of the MBG 0-1 group.

After the multivariate logistic regression models were adjusted to fragmented QRS [OR: 6.5 (1.4-28.6), $\mathrm{p}=$ 0.01], ST segment resolution at one hour [OR: $0.30(0.14-0.67), \mathrm{p}=0.003]$, TIMI frame number [OR: 1.05 (1.01-1.09), $\mathrm{p}=0.004]$, age, gender and pain-to-balloon time in patients who underwent PPCI, myocardial flushing was found to have deteriorated (as shown in Table 3).

\section{Discussion}

To our knowledge, this study is the first to evaluate the relationship between $\mathrm{f}-\mathrm{QRS}$ and microvascular dysfunction in inferior MI patients who underwent successful PPCI. In this study, the presence of f-QRS was demonstrated in patients with impaired microvascular perfusion despite normal TIMI flow. Multivariate logistic regression models revealed that fragmented QRS is the development of myocardial blushing.

Fragmented QRS represents myocardial scarring and has been found to be associated with poor prognostic outcomes in heart disease (5,19-22). Das et al. found that all causes of death and cardiac events were higher in patients with fragmented QRS compared to patients with non-fragmented QRS (5). In addition, a recent meta-analysis showed that initially fragmented QRS was associated with increased all-cause deaths in heart failure patients with reduced ejection fraction (23). In our study, we found that ejection fraction was lower in patients with fragmented QRS. Ejection fraction is an important parameter in determining the short- and long-term prognosis. 
In this study, fragmented QRS after angioplasty was associated with microvascular dysfunction in patients with acute inferior myocardial infarction whose infarct-related artery is the right coronary artery, as evidenced by angiographic results. Impaired microvascular perfusion was significantly associated with adverse cardiovascular events and mortality in patients with acute myocardial infarction. The success of primary PCI in myocardial infarction is assessed by different methods such as ST segment resolution, TIMI flow, and MBG. These microvascular perfusion markers represent a better predictor of clinical outcomes $(12,13)$. In this study, we used ST segment resolution and MBG to assess the success of reperfusion therapy. We found that impaired microvascular reperfusion was higher in patients with fragmented QRS. Similarly, previous studies have shown a negative relationship between fragmented QRS and reperfusion parameters $(14,15,16)$. In addition, Sridharan et al. found that fragmented QRS predicted impaired microvascular myocardial perfusion as assessed by ST segment resolution (17). In another study, Fatma et al. found that the presence of fragmented QRS in patients with myocardial infarction was associated with inadequate myocardial reperfusion, and they recommended its use as a simple, non-invasive parameter to evaluate myocardial perfusion (18).

Microvascular dysfunction is responsible for morbidity and mortality in many different cardiovascular diseases. Fibrosis is caused by irreversible damage to the cellular elements of the microvascular bed that feed the myocardium. This leads to delays in interventricular conduction. Thus, it may cause fragmented QRS formation. Therefore, it is possible that f-QRS represent impulse conduction abnormalities and create a setting for malignant reentrant ventricular arrhythmias and death. Das et al. showed that f-QRS in patients with coronary artery disease was associated with ventricular conduction delay due to myocardial scar detected by myocardial single photon emission tomography (24). In addition, in patients with known coronary artery disease, f-QRS was associated with major adverse cardiac events and was demonstrated as an independent predictor for all-cause mortality, and f-QRS was found to be associated with significantly higher arrhythmic events (25). We can say that the f-QRS seen on the ECG represents the conduction delay caused by the inhomogeneous activation of the ventricles due to myocardial scar.

\section{Limitations of the Study}

This study had a few limitations. The sample size was relatively small and cardiac complications had a short follow-up time (only hospital stay). In addition, the MBG assessment is not quantitative and is a visual assessment method.

\section{Conclusion}

Our study showed that the presence of f-QRS after successful PPCI was associated with microvascular dysfunction in patients with inferior MI. Fragmented QRS in standard ECGs can be used for risk assessment in myocardial infarction. More studies are needed to demonstrate the relationship between fragmented QRS and reperfusion parameters in myocardial infarction.

\section{Conflict of Interest Statement}

There is no conflict of interest.

\section{Financing}

The authors received no financial support.

\section{References}

1. Matthew T Roe, E.Magnus Ohman, Arthur C.P Maas, Robert H Christenson, Kenneth W Mahaffey, Christopher B Granger, et al. Shifting the open-artery hypothesis downstream: the quest for optimal reperfusion. J Am Coll Cardiol 2001; 37:9-18.

2. Martina Perazzolo Marra,Francesco Corbetti ,Luisa Cacciavillani, PGiuseppeTarantini, Angelo BrunoRamondo,MassimoNapodano,et al. Relationship between myocardial blush grades, staining, and severe microvascular damage after primary percutaneous coronary intervention a study performed with 
contrast-enhanced magnetic resonance in a large consecutive series of patients. Am Heart J 2010; 159:1124-1132.

3. Christopher E. Buller, Yuling Fu, Kenneth W. Mahaffey, Thomas G. Todaro, Peter Adams, Cynthia M. Westerhout, et al. ST-segment recovery and outcome after primary percutaneous coronary intervention for ST-elevation myocardial infarction: insights from the Assessment of Pexelizumab in Acute Myocardial Infarction (APEX-AMI) trial. Circulation 2008; 118:1335-1346.

4. Mithilesh Kumar Das, Chandan Saha, Hicham El Masry, Jonathan Peng, Gopi Dandamudi, Jo Mahenthiran, et al. Fragmented QRS on a 12-lead ECG: a predictor of mortality and cardiac events in patients with coronary artery disease. Heart Rhythm. 2007; 4:1385 \pm 92 .

5. Mithilesh Kumar Das, Waddah Maskoun, Changyu Shen, Mark A. Michael, Hussam Suradi, Mona Desai, et al. Fragmented QRS on twelve-lead electrocardiogram predicts arrhythmic events in patients with ischemic and nonischemic cardiomyopathy. Heart Rhythm. 2010; 7:74 \pm 80 .

6. Mithilesh K. Das, Mark A. Michael, Hussam Suradi, Jonathan Peng, Anjan Sinha, Changyu Shen, Jo Mahenthiran, et al. Usefulness of fragmented QRS on a 12-lead electrocardiogram in acute coronary syndrome for predicting mortality. Am J Cardiol 2009; 104: 1631-7.

7. Grzegorz Pietrasik, Ilan Goldenberg, Joanna Zdzienicka, Arthur J. Moss, Wojciech Zareba. Prognostic significance of fragmented QRS complex for predicting the risk of recurrent cardiac events in patients with Q-wave myocardial infarction. Am J Cardiol 2007; 100: 583-6.

8. Hafeez Ul Hassan Virk, Salman Farooq, Ali Raza Ghani, and Shilpkakumar Arora. QRS fragmentation: its role in sherlocking the arrhythmogenic heart. Journal of community hospital internal medicine perspectives 2016, 6: 31235.

9. Arnoud W.J. van 't Hof, Aylee Liem,Harry Suryapranata, Jan C.A. Hoorntje, MD;Menko-Jan de Boer, Felix Zijlstra. Angiographic assessment of myocardial reperfusion in patients treated with primary angioplasty for acute myocardial infarction: myocardial blush grade. Circulation. 1998; 97(23):23022306.

10. The TIMI study group. The thrombolysis in myocardial infarction(TIMI) trial: phase I findings.N Engl J Med. 1985;312(14):932-936.

11. Gensini G. G. A more meaningful scoring system for determining the severity of coronary heart disease. The American Journal of Cardiology . 1983;51(3):p. 606. doi: 10.1016/s0002-9149(83)80105-2.

12. Gibson CM, Cannon CP, Murphy SA, Ryan KA, Mesley R, Marble SJ, et al. Relationship of TIMI myocardial perfusion grade to mortality after administration of thrombolytic drugs. Circulation 2000; 101:125-130.

13. Henriques JP, Zijlstra F, van't Hof AW, de Boer MJ, Dambrink JH, Gosselink M, et al. Angiographic assessment of reperfusion in acute myocardial infarction by myocardial blush grade. Circulation 2003; 107:2115-2119.

14. Firat Ozcan, Osman Turak, Ugur Canpolat, Iskender Kadife, Sedat Avci, Ahmet Isleyen et al. Myocardial tissue perfusion predicts the evolution of fragmented QRS in patients with ST-segment elevation myocardial infarction undergoing primary percutaneous coronary intervention: fQRS and MBG in STEMI. Ann Noninvasive Electrocardiol. 2014;19:454e461.

15. Mustafa Cetin, Sinan Altan Kocaman, Tuncay Kiris, Turan Erdogan, Aytun Canga, Murtaza Emre Durakoglugil, et al. Absence and resolution of fragmented QRS predict reversible myocardial ischemia with higher probability of ST segment resolution in patients with ST segment elevation myocardial infarction. Korean Circ J. 2012;42:674.

16. Xianghong Ma, Wenting Duan, Pradeep Poudel, Junwei Ma, Deepak Sharma, Yanmin Xu. Fragmented QRS complexes have predictive value of imperfect ST-segment resolution in patients with STEMI after primary percutaneous coronary intervention. Am J Emerg Med. 2016;34:398e402.

17. Sridharan Umapathy, Rakesh Yadav, Kewal C. Goswami, Vinay K. Bahl. Prognostic significance of fragmented QRS in patients with ST-elevation myocardial infarction undergoing revascularization. Indian Heart Journal 2018; S126eS132.

18. Fatma Hizal Erdem, Yusuf Tavil, Huseyin Yazici, Nazif Aygul, Adnan Abacı,Bülent Boyacı. Association of Fragmented QRS Complex with Myocardial Reperfusion in Acute ST-Elevated Myocardial 
Infarction. Ann Noninvasive Electrocardiol 2013;18(1):69-74.

19. Xu, Y., Qiu, Z., Xu, Y.,Y. Xu., H. Bao., S. Gao \& X.ChengThe role of fQRS in coronary artery disease. A meta-analysis of observational studies. Herz, 2015:40(Suppl. 1), 8-15.

20. Pattara Rattanawong, Tanawan Riangwiwat,Narut Prasitlumkum, Nath Limpruttidham , Napatt Kanjanahattakij, Pakawat Chongsathidkiet, et al.Baseline fragmented QRS increases the risk of major arrhythmicevents in Brugada syndrome: Systematic review and meta-analysis.Annals of Noninvasive Electrocardiology,2017: 23(2), e12507.

21. Hamidreza Bonakdar, Hassan Moladoust, Jalal Kheirkhah, Esmat Abbaspour, Mohammad Assadian Rad, Arsalan Salari, et al. Significance of a fragmented QRS complex in patients with chronic total occlusion of coronary artery without prior myocardial infarction. Anatolian Journal of Cardiology 2016: 16(2), 106-112.

22. Pattara Rattanawong, Tanawan Riangwiwat, Chanavuth Kanitsoraphan, Pakawat Chongsathidkiet, Napatt Kanjanahattakij, Wasawat Vutthikraivit, et al. Baseline fragmented QRS increases the risk of major arrhythmic events in hypertrophic cardiomyopathy: Systematic review and meta-analysis. Annals of Noninvasive Electrocardiology, 2018:23(4), e12533.

23. Chanavuth Kanitsoraphan, Pattara Rattanawong, Poemlarp Mekraksakit, Pakawat Chongsathidkiet, Tanawan Riangwiwat, Napatt Kanjanahattakij,et al.Baseline fragmented QRS is associated with increased all-cause mortality in heart failure with reduced ejection fraction: A systematic review and meta-analysis Ann Noninvasive Electrocardiol. 2018;e12597.

24. Mithilesh K. Das, Bilal Khan, Sony Jacob, Awaneesh Kumar, Jo Mahenthiran. Significance of a fragmented qrs complex versus a q wave in patients with coronary artery disease. Circulation. 2006; 113: 2495-2501.

25. Mithilesh Kumar Das, Chandan Saha, Hicham El Masry, Jonathan Peng, Gopi Dandamudi, Jo Mahenthiran, et al. Fragmented qrs on a 12-lead ecg: A predictor of mortality and cardiac events in patients with coronary artery disease. Heart Rhythm. 2007; 4: 1385-1392.

\section{Hosted file}

fQRS table.pdf available at https://authorea.com/users/372027/articles/492754-relationshipbetween-fragmented-qrs-and-microvascular-function-in-patients-with-inferior-myocardialinfarction 